\title{
Genome sequence of OXA-23 producing Acinetobacter baumannii IHIT7853, a carbapenem-resistant strain from a cat belonging to international clone IC 1
}

\author{
Christa Ewers $^{1 *}$, Peter Klotz ${ }^{1}$, Sandra Scheufen ${ }^{1}$, Ursula Leidner ${ }^{1}$, Stephan Göttig ${ }^{2}$ and Torsten Semmler ${ }^{3}$
}

\begin{abstract}
Background: Multidrug resistance in Acinetobacter baumannii has dramatically increased in recent years worldwide. Thus, last-line antibiotics like carbapenems are increasingly being used which in turn further augments selection pressure for resistant strains. Resistance to carbapenems in A. baumannii is frequently mediated by carbapenemases, particularly OXA-23 and OXA-58. Carbapenemase-producing bacteria are mainly described in human patients and the intestinal tract represents a common source for such pathogens. In this study, we sequenced and analyzed the genome of A. baumannii IHIT7853, a carbapenem-resistant, OXA-23 producing strain isolated from cystitis in a cat in 2000 in Germany.

Results: Phylogenetic analysis revealed that IHIT7853 belonged to the globally distributed international clone IC1 and MLST type ST1/ST231 (Pasteur/Oxford MLST scheme). A phylogenetic tree based on the maximum common genome of 18 A. baumannii isolates placed IHIT7853 close to human clinical isolates, such as the multidrug-resistant (MDR) outbreak strain AYE that was isolated from a patient with pneumonia and cystitis in 2001 in France. The OXA-23 plasmid sequence could be determined as 53,995 bp in size, possessing resistance genes str $A$ and strB in addition to bla OXA-23.

Conclusions: The analysis of the genome of IHIT7853 reveals that companion animals carry MDR A. baumannii that resemble relevant clonal lineages involved in severe infections in humans. As urinary tract infections are often caused by bacteria that reside in the intestinal tract, future studies should unveil, if the animal gut serves as a source for MDR A. baumannii.
\end{abstract}

Keywords: OXA-23, Acinetobacter, Animal, Carbapenemase

\section{Background}

Acinetobacter baumannii is widely recognized as a nosocomial pathogen that is characterized by its intrinsic and acquired antimicrobial resistance. Of particular concern in hospital settings is the development of multidrugresistant (MDR) and particularly of carbapenem-resistant strains, which have increased markedly in recent years [1]. The intestinal microbiota forms a major reservoir of

\footnotetext{
*Correspondence: christa.ewers@vetmed.uni-giessen.de ${ }^{1}$ Institute of Hygiene and Infectious Diseases of Animals, Justus-LiebigUniversity Giessen, Frankfurter Str. 85-89, 35392 Giessen, Germany Full list of author information is available at the end of the article
}

A. baumannii and various infections, including bacteraemia, cystitis and wound infections can occur once the bacteria translocate across the intestinal barrier or reach the patient's skin as a consequence of faecal shedding [2, 3]. The finding of identical strains in the blood and gastrointestinal tract of patients with A. baumannii bloodstream infections strengthens the role of the gut as an important source of the pathogen [2].

Carbapenem resistance in A. baumannii is most frequently mediated through intrinsic (OXA-51-like) or acquired (e.g. OXA-23, OXA-58) carbapenem-hydrolyzing class $\mathrm{D} \beta$-lactamases (CHDLs) that confer resistance 
once they are overexpressed [4]. OXA-23-producing $A$. baumannii contribute to hospital-outbreaks worldwide and have recently tended to replace OXA-58-producing strains in some countries [1,5-8]. In Germany, the first outbreak of $A$. baumannii carrying the carbapenemase OXA-23 was observed in 2006 and nowadays it accounts for about $80 \%$ of MDR A. baumannii in Germany [7, 9]. Until recently, carbapenem-resistant $A$. baumannii have been mainly described in human patients. However, previous studies have identified this species as an emerging pathogen particularly in hospitalized companion animals and horses [9-11]. Here, we report the genome sequence of the carbapenem-resistant $A$. baumannii strain IHIT7853 that was isolated from the urine of a hospitalized cat with cystitis.

\section{Methods}

Bacteria, DNA isolation, genetic relatedness and antimicrobial susceptibility testing

Acinetobacter baumannii strain IHIT7853 was isolated from the urine of a cat admitted to a veterinary clinic in November 2000. For genomic DNA extraction late log-phase cells were harvested and lysed with EDTA, lysozyme, and detergent treatment, followed by proteinase $\mathrm{K}$ and RNase digestion using the DNeasy Blood \& Tissue Kit (Qiagen, Germany) according to the manufacturer's recommendation. Genomic DNA yield, purity, and concentration was evaluated using $0.7 \%$ agarose gel electrophoresis with $\lambda$-Hind III DNA marker (Thermo Fisher Scientific, USA). The genomic DNA was stored at $-20{ }^{\circ} \mathrm{C}$ until use. Genetic relatedness of $A$. baumannii isolates was investigated by semi-automated repetitive element palindromic (rep)-PCR using the DiversiLab ${ }^{\circledR}$ strain typing platform (BioMérieux, Nürtingen, Germany) as previously described [7]. Results were utilized with the DiversiLab ${ }^{\circledR}$ software using Pearson correlation to determine distance matrices and the unweighted pair group method with arithmetic averages to create dendrograms. MIC data were generated using the VITEK 2 system and AST-GN38 testing cards (BioMérieux).

\section{Genome and plasmid sequencing and annotation}

Whole-genome sequencing of $A$. baumannii strain IHIT7853 was performed on an Illumina MiSeq (MiSeq Reagent Kit v3, Illumina Inc.) resulting in 300 bp pairedend reads and an average coverage of $130 \times$. De novo assembly after quality trimming of the reads was conducted using CLC Genomics Workbench v. 9 (CLC bio, Denmark) with standard parameters, scaffolding and exclusion of contigs smaller than $200 \mathrm{bp}$. The genome was annotated using the Rapid Annotation using Subsystem Technology (RAST) server [12].
Functional classification of the genes was conducted by BLASTP with the Clusters of Orthologous Groups of proteins (COG) database [13]. Prediction of phage sequences and clustered regularly interspaced short palindromic repeats (CRISPRs) was performed with PHAST [14] and CRISPRfinder [15]. Antimicrobial resistance genes and insertion sequences were identified using the CGE website [16] and ISfinder, respectively [17].

Strain IHIT7960 was isolated from a dog in the same clinic where IHIT7853 has been obtained, and proved to be a natural plasmid-free, isogenic variant of A. baumannii IHIT7853. IHIT7960 was whole genome sequenced and de novo assembled as mentioned before. Whole genome sequencing reads from the plasmid-containing strain IHIT7853 were mapped against the genome of IHIT7960 and all reads that could not be mapped, and therefore represent plasmid DNA, were de novo assembled as described above. Few reads that could not be mapped during de novo assembly but did not represent plasmid sequences were not used to generate the plasmid contig.

\section{Phylogenetic analysis}

For comparative genomic analysis, genome sequences of representative $A$. baumannii strains were downloaded from the NCBI website: ATCC 17978 (CP012004), LAC-4 (CP007712), ABBL102 (LLHC00000000), TYTH-7 (AGSV00000000), GTC 03328 (BBNI00000000), RUH1486 (JZBU00000000), AB030 (CP009257), MDR-ZJ06 (CP001937), MDR-TJ (CP003500), NCGM 237 (AP013357), NIPH 1669 (APOQ00000000), AB307-0294 (NC_011595), strain A1 (CP010781), AYE (CU459141.1), AB0057 (NC_011586.1), TTU2014-131AME (LKJZ00000000), and FDAARGOS_123 (LORJ00000000). The source of the strains is given in Fig. 3. MLST types were determined following the Institute Pasteur [18] and Oxford [19] approach and using the CGE server [16]. The determination of the maximum common genome (MCG) alignment was done comprising those genes present in all 18 downloaded genomes [20]. For this purpose, we clustered the coding sequences based on the parameters sequence similarity (min. $70 \%$ ) and coverage (min. $90 \%$ ) and defined the genes that were present in each genome, thereby fulfilling the threshold parameters as MCG. This resulted in 2480 orthologous genes which we used for comparisons. Allelic variants of these genes from all genomes were then extracted by a BLAST-based approach, aligned individually for each gene and concatenated. This resulted in an alignment of $2.157 \mathrm{Mbp}$ for the $18 \mathrm{~A}$. baumannii strains. The alignment was used to generate a phylogenetic tree with RAxML 8.1 [21] using a General Time Reversible model and gamma correction for among site rate variation. 


\section{Quality assurance}

Acinetobacter baumannii IHIT7853 that was obtained from a single colony was maintained at the Institute of Hygiene and Infectious Diseases, Giessen, Germany, and genomic DNA was extracted from a pure culture. The 16S rDNA from the draft genome was used to check for contamination. GyrB gene sequencing and VITEK 2 biochemical identification (BioMérieux, Nürtingen, Germany) confirmed that the strain IHIT7853 belonged to the species A. baumannii. Possible contamination with other genomes and misassemblies were checked by mapping the reads back to the contigs. The read mapping of the draft genome of IHIT7853 is in the range of expected size distribution and the coverage of the reads was consistent throughout the genome.

\section{Results and discussion}

\section{General features}

Filtered $2.56 \mathrm{M}$ clean reads were assembled into scaffolds, and corresponding 130-fold coverage of the genome was generated. The draft genome sequence of $A$. baumannii IHIT7853 was 4,221,000 bp in size and had a $\mathrm{G}+\mathrm{C}$ content of $39.29 \%$ in 372 contigs, with N50 spanning 223,827 bp (Table 1). Annotation of this assembly identified 3960 coding sequences (CDSs), 65 tRNAs, and 12 rRNAs.

The reads belonging to the plasmid could be assembled into one single contig of 53,995 bp in size (Table 2; Fig. 1). The plasmid sequence had a $\mathrm{G}+\mathrm{C}$ content of $36.5 \%$ and revealed 53 protein coding sequences.

We categorized 3224 genes into COGs functional groups, including putative or hypothetical genes and genes of unknown function. For COGs distribution, abundant categories were amino acid transport and metabolism (261 ORFs), transcription factors (258 ORFs), general function prediction only (253 ORFs), and translation, ribosomal structure and biogenesis (231 ORFs) (Fig. 2a). The subsystem distribution and general

Table 1 General features of the A. baumannii IHIT7853 genome

\begin{tabular}{ll}
\hline Item & Value \\
\hline Number of contigs & 372 \\
Total contig length (bp) & $4,220,991$ \\
Fold coverage $(\mathrm{x})$ & 130 \\
$\mathrm{~N} 50$ (bp) & 223,827 \\
$\mathrm{G}+\mathrm{C}$ content (\%) & 39.29 \\
Number of protein coding genes & 3960 \\
Number of predicted transfer RNAs & 65 \\
Number of predicted ribosomal RNAs & 12 \\
GenBank accession number & LWTH00000000 \\
\hline
\end{tabular}

Table 2 General features of the pOXA-23-IHIT7853 plasmid

\begin{tabular}{ll}
\hline Item & Value \\
\hline Number of contigs & 1 \\
Total contig length (bp) & 53,995 \\
Fold coverage (x) & 450 \\
G + C content (\%) & 36.5 \\
Number of protein coding genes & 53 \\
GenBank accession number & KX118105 \\
\hline
\end{tabular}

information on the potential functional distribution of A. baumannii IHIT7853 is illustrated in Fig. 2b. Genes associated with amino acids and derivatives (466 ORFs), carbohydrates (340 ORFs), cofactors, vitamins, prosthetic groups, and pigments (264 ORFs), and protein metabolism (252 ORFs) were abundant among the SEED subsystem categories.

Five prophage regions have been identified in the genome of IHIT7853, of which 2 regions are intact and 3 regions are incomplete. Intact phages are (i) phi CTX, a P2-like cytotoxin-converting phage of Pseudomonas aeruginosa (Acc-No. NC_003278) of $34.1 \mathrm{~kb}$ in length and a G $+\mathrm{C}$ content of $38.72 \%$ and (ii) Acinetobacter phage YMC/09/02/B1251_ABA_BP (NC_019541), which is $45.36 \mathrm{~kb}$ in length, reveals a $\mathrm{G}+\mathrm{C}$ content of $39.05 \%$ and has previously been demonstrated to cause lysis of an OXA-23-producing carbapenem-resistant $A$. baumannii isolate from a septic patient [22]. One CRISPR candidate was detected consisting of 28 bp-repeat-areas and 54 spacers. Located downstream of the repeat and spacer region are the cas genes csy4-csy1, cas3, and cas1. Several insertion sequence (IS) elements, including ISAba1, ISAba12, IS15, IS17, and IS26 were identified using IS Finder.

The bla $_{\mathrm{OXA}-23}$ gene of $A$. baumannii IHIT7853 was localized on a $53.99 \mathrm{~kb}$ plasmid that also encoded aminoglycoside resistance genes $s t r A$ and $s t r B$. Other resistance genes detected in the genome of IHIT7853 were aminoglycoside resistance genes aadA1, aph(3') and aac(3)Ia, $\beta$-lactamase gene $b l a_{\text {OXA-69 }}$ (lacking an upstream insertion sequence), phenicol resistance gene catA1, and the genes sull and tet( $A$ ) coding for sulfonamide and tetracycline resistance, respectively. In agreement with these genetic features, high MICs were identified for imipenem $(\geq 16 \mathrm{mg} / \mathrm{L})$, piperacillin $(\geq 16 \mathrm{mg} / \mathrm{L})$, cefpirome $(\geq 64 \mathrm{mg} / \mathrm{L})$, gentamicin $(\geq 16 \mathrm{mg} / \mathrm{L})$, tetracycline $(\geq 16 \mathrm{mg} / \mathrm{L})$, and trimethoprim/sulfamethoxazole $(\geq 32 \mathrm{mg} / \mathrm{L})$. MICs to enrofloxacin $(2 \mathrm{mg} / \mathrm{L})$ and marbofloxacin $(\geq 4 \mathrm{mg} / \mathrm{L})$ are in correspondence with the finding of a single mutation in the gyrA sequence, resulting in an amino acid change from serine to leucine at position 83. 


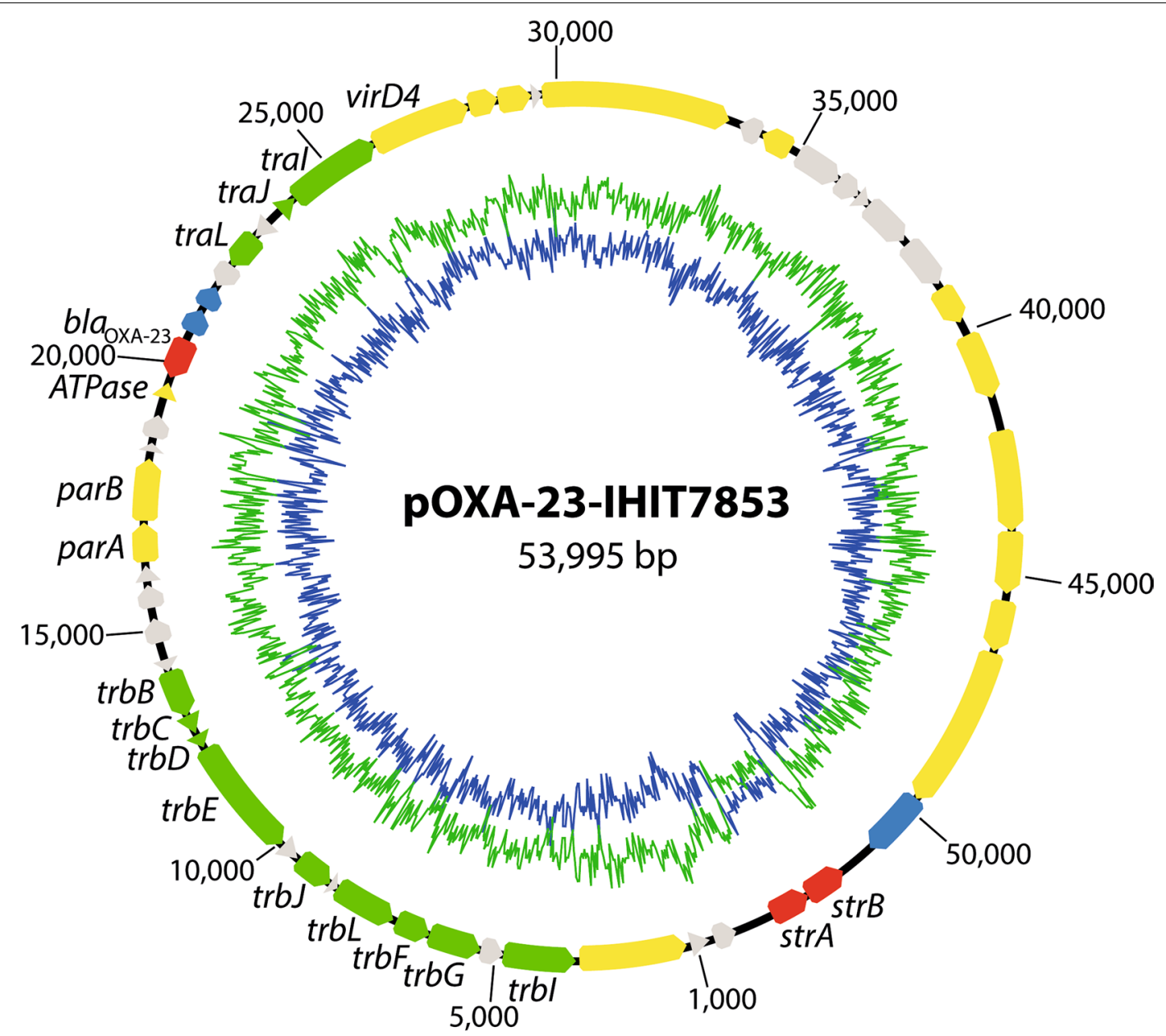

Fig. 1 Circular map of plasmid pOXA-23-IHIT7853 of A. baumannii strain IHIT7853 generated with Geneious 8.1.3 (Biomatters Ltd, Auckland, New Zealand). Broad arrows in the outer circle indicate genes coding for resistance factors (red), transfer proteins (green), hypothetical proteins (grey), insertion sequences/mobile elements (b/ue), and other factors (yellow), as partially indicated in the figure. Inner circles represent $\mathrm{G}+\mathrm{C}$ content (blue) and AT graph (green)

\section{Phylogenetic analysis}

Acinetobacter baumannii strain IHIT7853 was determined as sequence type $\mathrm{ST} 1^{\mathrm{P}}$ and $\mathrm{ST} 231^{\mathrm{Ox}}$ according to the Pasteur and Oxford MLST scheme, respectively. Genetic relatedness of IHIT7853 to other carbapenem-resistant $A$. baumannii clinical isolates which were collected during a 12-year period was analyzed by semi-automated rep-PCR. IHIT7853 showed highest similarities to two Oxa-23 positive isolates from human patients which were isolated from rectal swab and an intraoperative gut wound swab, respectively (data not shown). Clustering analysis showed that IHIT7853 belongs to the international clone IC1 in accordance with the fact that ST1 strains are member of this lineage. Together with IC2, this clonal lineage accounts for most of the nosocomial and communityacquired infections with $A$. baumannii worldwide [7]. Based on genomes from published A. baumannii strains from humans and on one genome available from cattle, we performed phylogenetic analysis on tree topology to identify genetic relatedness of the strains. IHIT7853 is highly similar to strain AYE, a MDR strain cultured from a patient with pneumonia and a urinary tract infection during an outbreak of ESBL- and VEB-1-producing A. baumannii isolates in a French hospital in 2001 (Fig. 3) [23]. Other human ST1/ST231 strains are also highly related to the animal strain suggesting that a transmission of such MDR strains may likely occur between animals and humans in both directions. 
a

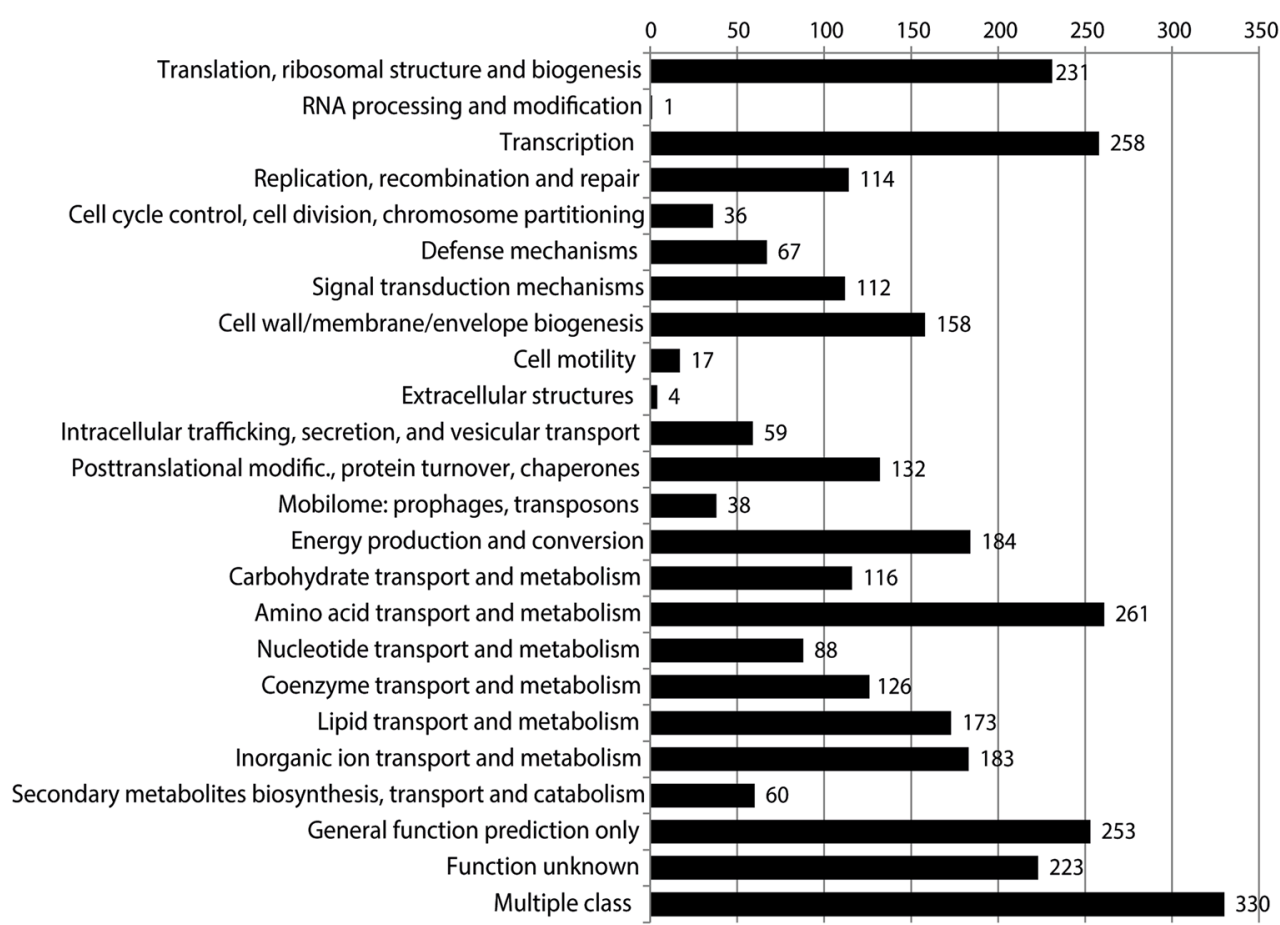

b

\section{Subsystem Coverage \\ Subsystem Category Distribution}
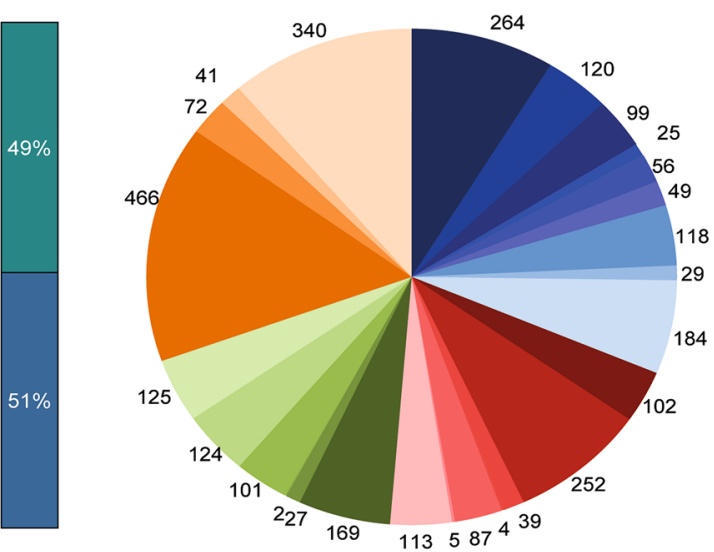

Subsystem Feature

Cofactors, Vitamins, Prosthetic Groups, Pigments (264) - Cell Wall and Capsule (120)

Virulence, Disease and Defense (99)

- Potassium metabolism (25)

Photosynthesis (0)

- Miscellaneous (56)

Phages, Prophages, Transposable elements, Plasmids (49)

Membrane Transport (118)

Iron acquisition and metabolism (29)

RNA Metabolism (184)

- Nucleosides and Nucleotides (102)

- Protein Metabolism (252)

- Cell Division and Cell Cycle (39)

- Motility and Chemotaxis (4)

- Regulation and Cell signaling (87)

Secondary Metabolism (5)

DNA Metabolism (113)

Fatty Acids, Lipids, and Isoprenoids (169)

Nitrogen Metabolism (27)

- Dormancy and Sporulation (2)

- Respiration (101)

Stress Response (124)

Metabolism of Aromatic Compounds (125)

- Amino Acids and Derivatives (466)

- Sulfur Metabolism (72)

Phosphorus Metabolism (41)

Carbohydrates (340)

Fig. 2 COG analysis and subsystem distribution. a COGs distribution of A. baumannii strain IHIT7853; b distribution of genes assigned to SEED subsystems (based on the RAST annotation server)

\section{Conclusions}

This is the first description of the genome and the OXA-23 plasmid sequence of a carbapenem-resistant A. baumannii from an animal. So far, whole genome studies of $A$. baumannii have nearly exclusively focused on comparing strains from human patients. Genome data of IHIT7853 revealed high relatedness of the cat strain to human clinical strains providing evidence for a possible transmission of these bacteria between humans and animals. Based on knowledge from human patients it should be considered that the gastrointestinal tract of animals might serve as a source of MDR $A$. baumannii probably leading to 


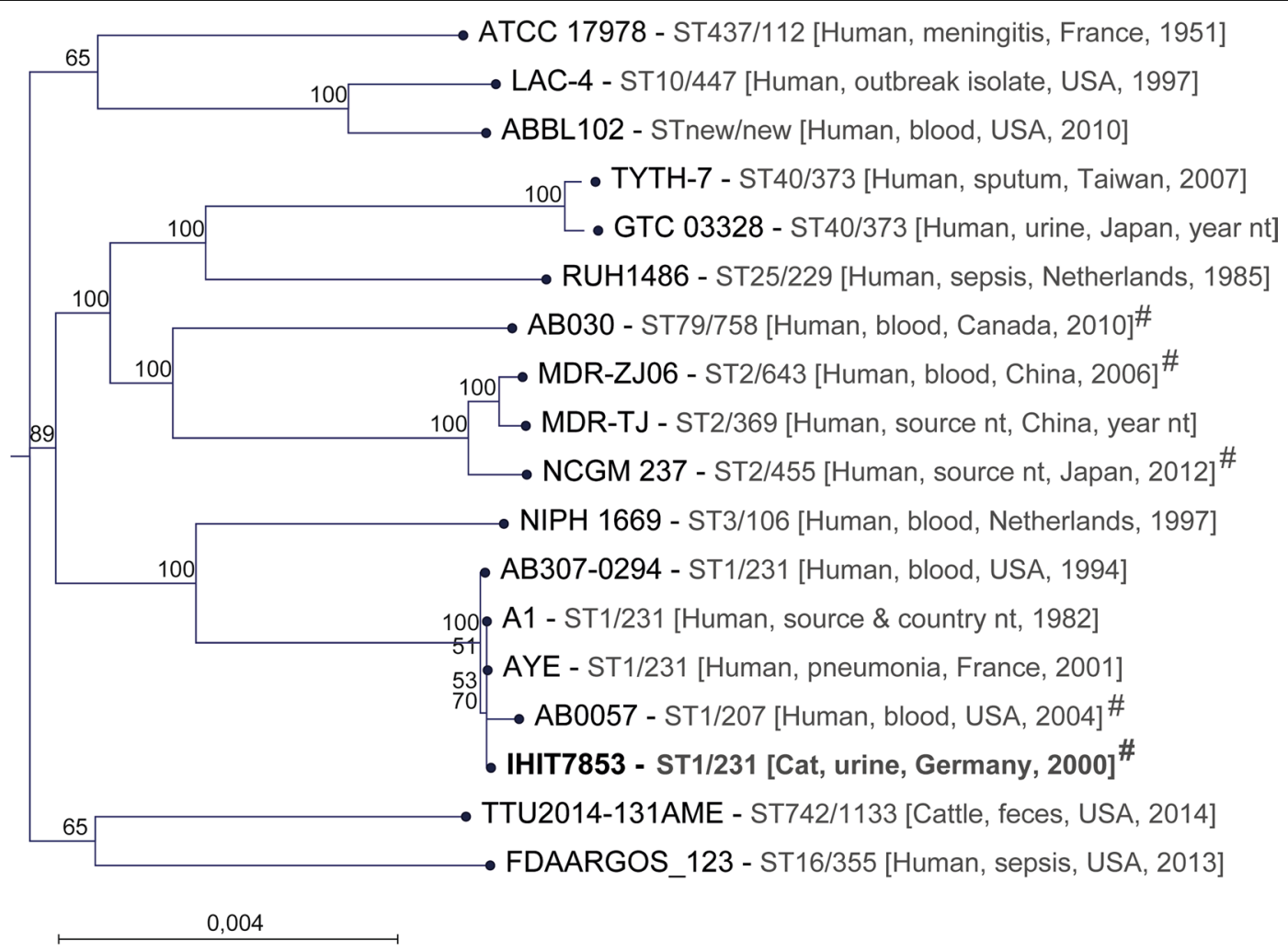

Fig. 3 Maximum likelihood tree (based on 2480 orthologous genes) of 18 A. baumannii strains. The scale indicates substitutions per site. Bootstrap values are based on 100 iterations. \# indicates the presence of a bla $a_{0 X-23}$ gene in the strains. The text in brackets provides information about host, sample material or clinical source, country, and year of isolation

infections at other sites [2, 3]. Future studies should unveil epidemiologic links between human and animal strains and track the genome dynamics in MDR A. baumannii from different hosts as well as from colonization and infection sites.

\section{Abbreviations}

COG: clusters of orthologous groups of proteins; CRISPR: clustered regularly interspaced short palindromic repeats; IC: international clone; IS: insertion sequence; ST: sequence type.

\section{Authors' contributions}

CE conceived, organized and supervised the project. CE, SG and TS interpreted the results, and edited the manuscript. SS and PK characterized the strains and maintained them in pure cultures. UL and SG performed antimicrobial susceptibility testing and rep-PCR analysis. UL prepared the high-quality genomic DNA, arranged the acquisition of sequence data and did the PFGE analysis. TS and CE performed the sequence assembly, gene prediction, gene annotation, analyzed the genome information, and drafted the manuscript. All authors read and approved the final manuscript.

\section{Author details}

${ }^{1}$ Institute of Hygiene and Infectious Diseases of Animals, Justus-Liebig-University Giessen, Frankfurter Str. 85-89, 35392 Giessen, Germany. ${ }^{2}$ Institute of Medical Microbiology and Infection Control, Hospital of the Johann Wolfgang von Goethe-University, Frankfurt am Main, Germany. ${ }^{3}$ Robert-Koch Institute, Berlin, Germany.

\section{Acknowledgements}

The authors are thankful to Petra Krienke and Inga Eichhorn for technical assistance during DNA sequencing.

\section{Competing interests}

The authors declare that they have no competing interests.

\section{Availability of supporting data}

The whole genome sequence of IHIT7853 and the sequence of pOXA-23IHIT7853 have been deposited at GenBank under the accession number LWTH00000000 and KX118105, respectively.

\section{Consent for publication}

Not applicable.

\section{Ethics approval}

Not applicable.

\section{Funding}

This study was financed by internal funding. PK was supported by grant from the Engemann Family Foundation.

Received: 14 June 2016 Accepted: 17 July 2016

Published online: 28 July 2016

\section{References}

1. Peleg AY, Seifert H, Paterson DL. Acinetobacter baumannii: emergence of a successful pathogen. Clin Microbiol Rev. 2008;21(3):538-82. 
2. Donskey CJ. Antibiotic regimens and intestinal colonization with antibiotic-resistant gram-negative bacilli. Clin Infect Dis. 2006;43:62-9.

3. Thom KA, Hsiao WW, Harris AD, Stine OC, Rasko DA, Johnson JK. Patients with Acinetobacter baumannii bloodstream infections are colonized in the gastrointestinal tract with identical strains. Am J Infect Control. 2010;38(9):751-3.

4. Poirel L, Naas T, Nordmann P. Diversity, epidemiology, and genetics of class D beta-lactamases. Antimicrob Agents Chemother. 2010;54(1):24-38.

5. Adams-Haduch JM, Paterson DL, Sidjabat HE, Pasculle AW, Potoski BA, Muto CA, Harrison LH, Doi Y. Genetic basis of multidrug resistance in Acinetobacter baumannii clinical isolates at a tertiary medical center in Pennsylvania. Antimicrob Agents Chemother. 2008;52(11):3837-43.

6. Corvec S, Poirel L, Naas T, Drugeon H, Nordmann P. Genetics and expression of the carbapenem-hydrolyzing oxacillinase gene blaOXA-23 in Acinetobacter baumannii. Antimicrob Agents Chemother. 2007;51(4):1530-3.

7. Higgins PG, Dammhayn C, Hackel M, Seifert H. Global spread of carbapenem-resistant Acinetobacter baumannii. J Antimicrob Chemother. 2010;65(2):233-8

8. Coelho JM, Turton JF, Kaufmann ME, Glover J, Woodford N, Warner M, Palepou MF, Pike R, Pitt TL, Patel BC, et al. Occurrence of carbapenemresistant Acinetobacter baumannii clones at multiple hospitals in London and Southeast England. J Clin Microbiol. 2006;44(10):3623-7.

9. Kohlenberg A, Brummer S, Higgins PG, Sohr D, Piening BC, de Grahl C, Halle E, Ruden H, Seifert H. Outbreak of carbapenem-resistant Acinetobacter baumannii carrying the carbapenemase OXA-23 in a German university medical centre. J Med Microbiol. 2009;58(Pt 11):1499-507.

10. Zordan S, Prenger-Berninghoff E, Weiss R, van der Reijden T, van den Broek P, Baljer G, Dijkshoorn L. Multidrug-resistant Acinetobacter baumannii in veterinary clinics, Germany. Emerg Infect Dis. 2011;17(9):1751-4.

11. Vaneechoutte $M$, Devriese LA, Dijkshoorn L, Lamote B, Deprez P, Verschraegen G, Haesebrouck F. Acinetobacter baumannii-infected vascular catheters collected from horses in an equine clinic. J Clin Microbiol. 2000;38(11):4280-1.

12. Aziz RK, Bartels D, Best AA, DeJongh M, Disz T, Edwards RA, Formsma K, Gerdes S, Glass EM, Kubal M, et al. The RAST Server: rapid annotations using subsystems technology. BMC Genom. 2008;9:75.
13. Tatusov RL, Fedorova ND, Jackson JD, Jacobs AR, Kiryutin B, Koonin EV, Krylov DM, Mazumder R, Mekhedov SL, Nikolskaya AN, et al. The COG database: an updated version includes eukaryotes. BMC Bioinformatics. 2003;4:41.

14. Zhou Y, Liang Y, Lynch KH, Dennis JJ, Wishart DS. PHAST: a fast phage search tool. Nucleic Acids Res. 2011;39(Web Server issue):W347-52.

15. Grissa I, Vergnaud G, Pourcel C. CRISPRFinder: a web tool to identify clustered regularly interspaced short palindromic repeats. Nucleic Acids Res. 2007;35(Web Server issue):W52-7.

16. The CGE (Center for Genomic Epidemiology) server. https://cge.cbs.dtu dk/services/MLST/. Accessed 12 May 2016.

17. Siguier P, Perochon J, Lestrade L, Mahillon J, Chandler M. ISfinder: the reference centre for bacterial insertion sequences. Nucleic Acids Res. 2006;34(Database issue):D32-6.

18. Diancourt L, Passet V, Nemec A, Dijkshoorn L, Brisse S. The population structure of Acinetobacter baumannii: expanding multiresistant clones from an ancestral susceptible genetic pool. PLoS One. 2010;5(4):e10034

19. Bartual SG, Seifert $H$, Hippler C, Luzon MA, Wisplinghoff $H$, RodriguezValera F. Development of a multilocus sequence typing scheme for characterization of clinical isolates of Acinetobacter baumannii. J Clin Microbiol. 2005;43(9):4382-90.

20. von Mentzer A, Connor TR, Wieler LH, Semmler T, Iguchi A, Thomson NR, Rasko DA, Joffre E, Corander J, Pickard D, et al. Identification of enterotoxigenic Escherichia coli (ETEC) clades with long-term global distribution. Nat Genet. 2014;46(12):1321-6.

21. Stamatakis A. RAxML version 8: a tool for phylogenetic analysis and postanalysis of large phylogenies. Bioinformatics. 2014;30(9):1312-3.

22. Jeon J, Kim JW, Yong D, Lee K, Chong Y. Complete genome sequence of the podoviral bacteriophage YMC/09/02/B1251 ABA BP, which causes the lysis of an OXA-23-producing carbapenem-resistant Acinetobacte baumannii isolate from a septic patient. J Virol. 2012;86(22):12437-8.

23. Poirel L, Menuteau O, Agoli N, Cattoen C, Nordmann P. Outbreak of extended-spectrum beta-lactamase VEB-1-producing isolates of Acinetobacter baumannii in a French hospital. J Clin Microbiol. 2003;41 (8):3542-7.

\section{Submit your next manuscript to BioMed Central and we will help you at every step:}

- We accept pre-submission inquiries

- Our selector tool helps you to find the most relevant journal

- We provide round the clock customer support

- Convenient online submission

- Thorough peer review

- Inclusion in PubMed and all major indexing services

- Maximum visibility for your research

Submit your manuscript at www.biomedcentral.com/submit
(O) BioMed Central 